\title{
MINIMALLY INVASIVE TRICUSPIDE VALVE SURGERY WITHOUT CAVAL OCCLUSION: SHORT AND MID-TERM RESULTS.
}

Vito Margari ${ }^{1}$, Pietro Malvindi ${ }^{1}$, Adriano De Santis ${ }^{1}$, Kounakis Giorgio ${ }^{1}$, Giuseppe

Visicchio $^{1}$, Giuseppe Mastrototaro ${ }^{1}$, Pierpaolo Dambruoso ${ }^{1}$, CARMINE CARBONE ${ }^{1}$, and Domenico Paparella ${ }^{1}$

${ }^{1}$ Ospedale Santa Maria

October 8, 2020

\begin{abstract}
Abstract Objectives: The use of minimally invasive or transcatheter interventions rather than standard full sternotomy operations to treat Tricuspid valve disease is increasing. Debate however is still open regarding venous drainage management during cardiopulmonary bypass and wheatear or not superior and inferior vena cava should be occluded during opening of the right atrium to avoid air entrance in the venous line. The aim of the present study is to report operative outcomes and mid-term follow-up results of minimally invasive tricuspid valve surgery performed without caval occlusion. Methods: This is a retrospective outcome evaluation from institutional records with prospective data entry. We searched for all the patients who underwent right mini-thoracotomy tricuspid valve surgery isolated or combined with mitral valve surgery during the period June 2013 February 2020. Results: During the study period 68 consecutive patients underwent minimally invasive tricuspid valve surgery without occlusion of cava veins. Survival at a 5-year and 8-year follow up was $100 \%$ and $79 \%$, respectively. At follow-up no patient had an NHYA class greater than two, only one patient was re-hospitalized for heart failure for an atrial fibrillation episode. One patient was hospitalized for a pericardiocentesis twenty days after discharge No severe tricuspid regurgitation was evident at echocardiographic follow up. Five patients had 2+ TR. Conclusion Our results show that performing tricuspid surgery without caval occlusion is safe. There is no clinical evidence of gas embolism. Mid-term follow up data confirm that minimally invasive approach does not alter the quality of surgery.
\end{abstract}

MINIMALLY INVASIVE TRICUSPIDE VALVE SURGERY WITHOUT CAVAL OCCLUSION: SHORT AND MID-TERM RESULTS.

Vito Margari ${ }^{1}$ MD, Pietro Giorgio Malvindi ${ }^{1}$ MD, PhD, Adriano De Santis ${ }^{1}$ MD, Giorgio Kounakis ${ }^{1}$ MD, Giuseppe Visicchio ${ }^{1}$ MD, Giuseppe Mastrototaro ${ }^{1}$, Pierpaolo Dambruoso ${ }^{1} \mathrm{MD}$, Carmine Carbone ${ }^{1}$ MD, Domenico Paparella ${ }^{1,2}$ MD.

${ }^{1}$ Santa Maria Hospital, Department of Cardiac Surgery. GVM Care \& Research, Bari, Italy

${ }^{2}$ Dipartimento Scienze Mediche e Chirurgiche, Università di Foggia, Italy

Conflict of interest and source of funding: None

2 figures, 4 Tables

Address for Correspondence:

Vito Margari, MD

Department of Cardiac Surgery 
Santa Maria Hospital. GVM Care and Research

Department of Cardiac Surgery

Via de Ferrariis 22, 70124

Bari, Italy

Tel.: +393927574382

Email: vitomargari@icloud.com

\section{Introduction}

The use of minimally invasive or transcatheter interventions rather than standard full sternotomy operations to treat heart valve disease is increasing. Management of tricuspid valve disease is no different: minimally invasive techniques are more frequently utilized to perform standardized surgical operations. Surgery through right mini-thoracotomy approach has established itself as an optimal option to treat pathologies affecting atrio-ventricular valves [1] [2]. Experienced and specifically trained surgeons can achieve excellent results as those obtained with standard sternotomy, with many studies reporting better pain control, faster recovery and a shorter hospital stay as compared to conventional surgery [3]. Excellent results have also been reported for tricuspid valve operations performed with a minimally invasive approach [4] [5] [6], debate however is still open regarding venous drainage management during cardiopulmonary bypass (CPB) and wheatear or not superior and inferior vena cava should be occluded during opening of the right atrium to avoid air entrance in the venous line. The aim of the present study is to report operative outcomes and mid-term follow-up results of minimally invasive tricuspid valve surgery performed without caval occlusion.

\section{Material and Methods}

\section{Population and study design}

This is a retrospective outcome evaluation from institutional records with prospective data entry and from a dedicated prospective institutional database regarding right mini-thoracotomy cardiac procedures. All patients sign an informed consent form to allow clinical and administrative data storage and utilization for scientific purposes according to the General Data Protection Regulation (GDPR). Because of the retrospective nature of this study, the local Ethic Committee waived the need for patient consent. We searched for all the patients who underwent right mini-thoracotomy tricuspid valve surgery isolated or combined with mitral valve surgery during the period June 2013 - February 2020. Primary outcomes of the study were postoperative mortality (30 days) and occurrence of postoperative complications. Secondary outcomes were survival and freedom from reoperation. The follow-up (median time 35 months) was $100 \%$ complete and accomplished by direct contact of patients or their next-of-kin, and included evaluation of the most recent echocardiogram, functional class and eventual hospital admissions.

\section{Definitions}

Definitions of the preoperative variables align with the notes about EuroSCORE II. Postoperative outcomes were recorded according to the VARC-2 criteria. Surgical indications were based on recommendations of the most recent guidelines (ACC/AHA, ESC/EACTS).

Definition of tricuspid valve regurgitation severity was based on the absolute size of the regurgitation jet by colour flow imaging, the relative size of the regurgitation jet ( relative to the right atrium area), and the hepatic venous flow pattern, as recommended by the guidelines of the American Society of Echocardiography.

Perioperative MI has been evaluated according to the universal definition of myocardial infarction [7].

\section{Preoperative assessment and surgical techniques}

Heart valves function was assessed by transthoracic and transoesophageal echocardiography. In the early years of our experience all patients underwent preoperative $\mathrm{CT}$ angiogram of the whole aorta to evaluate 
femoral access, aorto-iliac axes and the size of aortic root and the ascending aorta. In the last four years, CT scan was performed only in case of redo procedures and clinical suspicion or evidence of severe atherosclerotic disease. Coronary arteries were assessed by coronary angiogram.

Most of the operations (93\%) on the atrioventricular valves in our institute were performed through right mini-thoracotomy (MICS). In some cases, median sternotomy was performed.

Main reasons for a sternotomy approach were: left ventricular ejection fraction $<25 \%$, presence of pleural adhesion for previous thoracic operations or chronic disease, severe chronic obstructive pulmonary disease, severe peripheral arterial disease, active endocarditis with abscess involving the mitro-aortic continuity [1].

Right anterior thoracotomy was performed through a 5-7 cm skin incision placed at 4 th intercostal space. In men, the incision is usually immediately above the nipple. In women with pronounced breasts the incision can be made in the sub-mammary sulcus. In the case of a small and not very pronounced breast in women and in all men, a periareolar incision can be made. Two trocars were used, one for the camera in the third intercostal space, and one for $\mathrm{CO} 2$ delivery in the fifth space.

At the beginning of our experience, the surgical operation was performed on direct vision and video assistance, while, recently, we have move towards a totally endoscopic procedure $(n=17,25 \%)$.

All interventions were performed with cardiopulmonary bypass with peripheral cannulation.

An incision of about $4 \mathrm{~cm}$ was performed at the level of the groin, with exposure of the femoral vessels. The femoral artery was cannulated using the Seldinger technique with a 17-19 Fr Bio-medicus cannula (Medtronics, Minneapolis, MN 55432-5604 USA) or a 22 EOPA cannula (Medtronics, Minneapolis, MN 55432-5604 USA). The choice of cannula was determined by the diameter and characteristics of the femoral artery. When an endo aortic balloon was used, the femoral artery was cannulated with a 21-23 Fr EndoReturn cannula (Edwards Lifesciences, Irvine, CA 92614 USA).

Aortic clamp and cardioplegia delivery was achieved by Endoaortic balloon or direct cross clamp of the ascending aorta with a Chitwood or a Cygnet vascular clamp. In the first scenario a Y shaped 21 or $23 \mathrm{~F}$ cannula was used. The decision between these two different techniques was based on the anatomy of the sinotubular junction, the aorto-iliac-femoral anatomy and chest conformation. Generally, all redo operations were performed using an endobaloon aortic clamp.

A double venous cannulation has been invariably used. A 14 Fr cannula (Medtronics, Minneapolis, MN 55432-5604 USA) was usually inserted percutaneously and with the Seldinger technique into the right internal jugular vein and advanced into the superior caval vein. Through the femoral vein a 23-25 Fr venous cannula (LivaNova, London, W2 6LG, United Kingdom) was positioned at the level of the inferior vena cava.

Positioning of the cannulas was guided by transoesophageal echocardiogram.

The tip of the cannula in the superior vena cava laid at superior atriocaval junction, while the tip of the cannula in the inferior vena cava $1 \mathrm{~cm}$ beyond the inferior atriocaval junction, in the right atrium.

Two types of pumps for cardiopulmonary bypass have been used: in the first part of the experience a roller pump has been used, subsequently a centrifugal pump has been used instead. In all interventions, before right atriotomy, an active venous drainage (vacuum) was used, the intensity of the venous drainage ranges from -20 to a maximum of $-40 \mathrm{mmHg}$.

In all cases caval veins were not snared nor occluded from the inside and the venous drainage was adjusted to avoid air suction while allowing a satisfactory exposure of the tricuspid valve. Cardiopulmonary bypass was generally conducted in normothermia with the use of normothermic blood cardioplegia. In case of redo procedures or use of endoaortic balloon, crystalloid custodial cardioplegia was used and mild systemic hypothermia was established (32degC).

Statistical analysis 
Patients' characteristics were presented as mean +- standard deviation, median [range] or as counts and percentages.

Survival and mid-term outcomes rates were calculated using the Kaplan-Meier method. Statistical analyses were performed using the Stat-View Statistical Software Package 5.0 (SAS Institute, Inc., Cary, NC, USA), NCSS 2001 (Number Cruncher Statistical System, Kaysville, Utah).

\section{Results}

During the study period 68 consecutive patients underwent minimally invasive tricuspid valve surgery. The mean age was $69+/-14$ years and $48(70 \%)$ were female. A redo operation was performed in 5 patients. Table 1 details preoperative patients' characteristics. Isolated tricuspid valve repair was performed in 4 patients, while in 64 cases it was associated with mitral valve surgery $+/$ - AF ablation.

In 17 cases (25\%), including the five patients who underwent a redo operation, an endoaortic balloon clamp was used; in the remaining 51 patients (75\%) an external cross clamp was applied. The tricuspid valve was repaired in all cases with a restrictive annuloplasty using a rigid ring (Table 2). In combined interventions mean CPB time was $156+/-43$ (minutes), mean cross clamp time was $118+/-32$ (minutes). In isolated TVr mean CPB and cross clamp time were $105+/-41$ and $74+/-6$ minutes respectively. Operative times decreased over the study period: the analysis of the 2-year interval periods showed that mean CPB time shortened from 186+-48 minutes in the period 2013-2014 to 127+-16 minutes during the period 2019-2020 (anova $\mathrm{p}=0.002$ ); mean cardioplegic arrest time decreased from $132+-28$ minutes in the first two years to 97+-13 minutes in the period 2019-2020 (anova $\mathrm{p}=0.02$ ).

Conversion to sternotomy was necessary in one case for uncontrolled bleeding from left atrial appendage, damaged by external aortic clamp. One patient was taken back to theatre for excessive bleeding that was ultimately controlled through the original right mini-thoracotomy access. A new perioperative cerebral stroke occurred in one patient; two patients sustained an episode of transient ischemic attack during the early postoperative course. The mean stay in ICU was $56+/-48$ hours and was prolonged for five patients who suffered low cardiac output syndrome requiring drug support and, in one case, renal replacement therapy with CVVHD. Cardiac enzymes were routinely evaluated during the first postoperative days (table 3), no patient sustained a perioperative myocardial infarction. In 25 cases $(37 \%)$ blood transfusion was performed during the post-operative course. (Table 3. Reports postoperative laboratory tests).

Three patients developed infection $(n=1)$ and lymphoceles $(n=2)$ with dehiscence of the inguinal wound; no complications were registered at the mini-thoracotomy site. All patients were successfully discharged after a mean hospital stay of $8.9+/-7.2$ days.

Survival at a 5-year and 8-year follow up was $100 \%$ and 79\%, respectively (Figure 1). (Table 4. Reports early postoperative outcomes). At follow-up no patient had an NHYA class greater than two, only one patient was re-hospitalized for heart failure for an atrial fibrillation episode. One patient was hospitalized for a pericardiocentesis twenty days after discharge

No severe tricuspid regurgitation was evident at echocardiographic follow up (Figure 2 ). Five patients had $2+\mathrm{TR}$.

\section{Discussion}

Minimal access cardiac surgery has undergone continuous development over the past twenty years and has become in certain settings the gold standard for the treatment of atrioventricular valve pathologies. Several institutional have reported outstanding results as a minimally invasive surgical approach provided to be safe and effective, and propensity matched studies showed comparable early and mid-term results between right mini-thoracotomy access and conventional sternotomy approach [8][9][10][11]. However, all these experiences collaterally highlighted that patients in sternotomy surgery cohorts had a higher preoperative risk score and a higher prevalence of redo procedures, rheumatic disease and mitral valve replacement. Furthermore, patients who underwent cardiac surgery through sternotomy were more likely to receive an associated procedure 
involving the tricuspid valve. Our experience suggest that the performance of tricuspid valve surgery through a minimally invasive approach is safe and effective.

In our practice, the reasons favouring a sternotomy approach were limited to the presence of pleural adhesions from previous thoracic operations, severe peripheral arterial disease, or active endocarditis with abscess involving the mitro-aortic continuity. During the study period, more than $95 \%$ of surgical operations on atrioventricular valves have been performed through a right mini-thoracotomy access and in $20 \%$ of the cases they included a combined procedure on mitral and tricuspid valves [12]. Recent guidelines recommend intervention for severe symptomatic tricuspid valve regurgitation at the time of left-side heart valves operation (ACC and ESC) and in case of severe primary tricuspid regurgitation in patients who are symptomatic despite an optimal medical therapy (ESC). There is still a big gap in knowledge regarding the benefit associated with tricuspid valve intervention and its timing, however, an untreated moderate/severe TR has been associated with a worse survival both in primary tricuspid valve regurgitation or in case of tricuspid disease secondary to mitral dysfunction. Tricuspid valve surgery has been increasingly performed during the last decade, however it is still associated with high early mortality (8-10\%) and morbidity rate and long period of hospitalisation [13]. A minimally invasive approach aiming a less invasive access and a faster recovery could be beneficial in this frail and comorbid patients but few reports in literature have focused on mini thoracotomy tricuspid valve surgery [14] [15].

Our data showed the minimally invasive approach has been safe and has shown good results. The times of mechanical ventilation and ICU stay are in line with the values reported in the literature. Although neurological complications are present in a small number of cases, 3 patients (4\%) are an element still present in valve surgery, in our experience they have mostly occurred with the use of endo-aortic clamping with endo-clamp. Despite the presence of a share of blood in the right atrium, the visualization of the tricuspid valve was optimal, the echocardiographic results at discharge and follow-up are satisfactory, shows an efficacy of the surgical technique performed.

External snaring of the caval veins is commonly performed before the opening of the right heart chambers. This manoeuvre can be hazardous through a right mini thoracotomy approach, especially in presence of frail tissue or in case of redo procedure as bleeding control can be challenging or impossible without conversion to sternotomy. Previous reports have underlined this technical issue and described alternative solutions of management of the venous drainage without the external snaring of the caval veins. Murzi et al. reported in 2009 their experience with a single two-stage femoral venous cannula able to provide a satisfactory drainage directly from the superior and inferior caval vein. In seventeen redo cases, this strategy allowed a satisfactory venous drainage while opening the right atrium without snaring the caval veins [16]. The same group confirmed these results on a larger scale reporting the safety and effectiveness of using a double-stage femoral venous cannula with two distal perforated sections separated by a nonperforated segment of $15 \mathrm{~cm}$ in length in right-minithoracotomy surgery requiring the opening of the right atrium without occluding the venae cavae [17]. A similar solution with a single-stage femoral venous cannula was described by Peng et al as they reported a successful CPB management and surgical repair of tricuspid valve in 8 patients who underwent redo surgery through a right minithoracotomy approach [18]. A different trick has been proposed in 2011 by Sansone et al. reporting an internal occlusion of the two caval veins by a Foley catheter in 3 redo cases of combined mitral and tricuspid valve surgery [19]. This technique has been subsequently developed by the same authors with the implementation of an endovascular balloon catheter [20]. Dandolu et al. described in 2005 the incidental observation that the opening of the right atrium without caval snaring did not lead to air entry in the venous reservoir. They further studied this finding on an animal model characterised by double venous cannulation and no caval veins occlusion and reported that, after the opening of the right atrium, the venous drainage remained satisfactory with a minimal entering of air in the superior vena cava [21].

In our experience no type of caval veins occlusion was used and all procedures were performed with the usual setting of double venous cannulation: surgical technique is simpler, there is a reduction in tissue dissection, especially in reoperations, reducing the chances of damage and bleeding. In mini-thorototomy the detection of bleeding and its repair can be complex. Venous drainage should be modulated to maintain a blood level 
in the right atrium below the tricuspidal valve plane. In order to guarantee the visualization of the tricuspid valve in all its parts we recommend that blood level should be approximately $1 \mathrm{~cm}$ below the septal leaflet. Through the venous cannulas with active drainage a mixture of air and continuous blood is guaranteed, this allows that an air column does not form without blocking the cardiopulmonary pump. The continuous passage of blood in the venous circuit pushes the air bubbles from the cannula to the reservouir. For this reason it is necessary to maintain a minimum level of blood in the right atrium. Generally the mixture of air and blood is managed by the active vacuum drainage, changing the sucking intensity. In the event that the amount of air is excessive and causes a temporary letup of the venous return, the surgeon can pull the draining tip of the cannula into the inferior vena cava. This shift allows an increase in blood drainage and a mobilization of air from the venous circuit to the reservoir.

Conclusions.

Our results show that performing tricuspid surgery without caval occlusion is safe. The air was captured by the active vacuum drainage system without causing damage. There is no clinical evidence of gas embolism. Vacuum-assisted drainage in centrifugal pump could cause an increase in wall stress on red blood cells but only in few cases perioperative hematuria occurred without clinical repercussions. Mid-term follow up data confirm that minimally invasive approach does not alter the quality of surgery.

\section{Tables}

Table 1. Preoperative patients' characteristics

\begin{tabular}{ll}
\hline Variables & Overall population n: $\mathbf{6 8}$ \\
\hline Age (years) & $69+/-14,07$ \\
Male/Female & $20 / 48$ \\
LVEF(\%) & $54+/-7$ \\
eGFR $<85 \mathrm{ml} / \mathrm{min}$ & $10(15 \%)$ \\
Haemodialysis & $3(3 \%)$ \\
Atrial Fibrillation & $48(63 \%)$ \\
Euro Score II\% & $3,1+/-2,16$ \\
PAPs $>30 \mathrm{mmHg}$ & $47(69 \%)$ \\
Reoperation & $5(7 \%)$ \\
\hline
\end{tabular}

LVEF ( Left Ventricular Ejection Fraction ), Paps ( Pulmonary Artery Pressure),

Table 2. Operative details

\begin{tabular}{ll}
\hline Variables & Overall Population n:68 \\
\hline Isolated TV repair & $4(5 \%)$ \\
MV Replacement + TV repair & $33(48 \%)$ \\
MV repair + TV repair & $31(46 \%)$ \\
AF ablation & $7(10 \%)$ \\
CPB time (min) & $156+/-43(\min )$ \\
Cardioplegic arrest time (min) & $118+/-32(\min )$ \\
CPB time isolated TV repair & $105+/-41(\min )$ \\
Cardioplegic arrest time (min) isolated TV repair & $74+/-6(\min )$ \\
Conversion to median sternotomy & $1(1,5 \%)$ \\
\hline
\end{tabular}

CPB ( Cardio Pulmonary Bypass )

Table 3. Postoperative laboratory tests 


\begin{tabular}{ll}
\hline Variables & Overall population $\mathbf{n : ~} \mathbf{6 8}$ \\
\hline Trop I POD1 ng/ml & $11,6+/-29$ \\
CK-Mb POD1 ng/ml & $48,7+/-55,2$ \\
AST POD1 & $66,18+/-36,3$ \\
ALT POD1 & $36,6+/-40,26$ \\
GGT POD1 & $30,4+/-46,8$ \\
\hline
\end{tabular}

POD ( Post Operative Day)

Table 4. Early postoperative outcomes

\begin{tabular}{ll}
\hline Variables & Overall population n: $\mathbf{6 8}$ \\
\hline Transient Ischemic Attack & $2(3 \%)$ \\
Cerebral stroke & $1(1,5 \%)$ \\
Re-exploration & $1(1,5 \%)$ \\
Low cardiac output sindrome & $4(6 \%)$ \\
Continous Veno-Venous HemoDialysis & $1(1,5 \%)$ \\
Groin complication & $3(4 \%)$ \\
Intensive care length of stay (hours) & $56+/-48$ \\
Hospital length of stay (days) & $8,9+/-7,2$ \\
Hospital mortality & 0 \\
\hline
\end{tabular}

Figure 1. Kaplan-Meier curve of survival after surgery

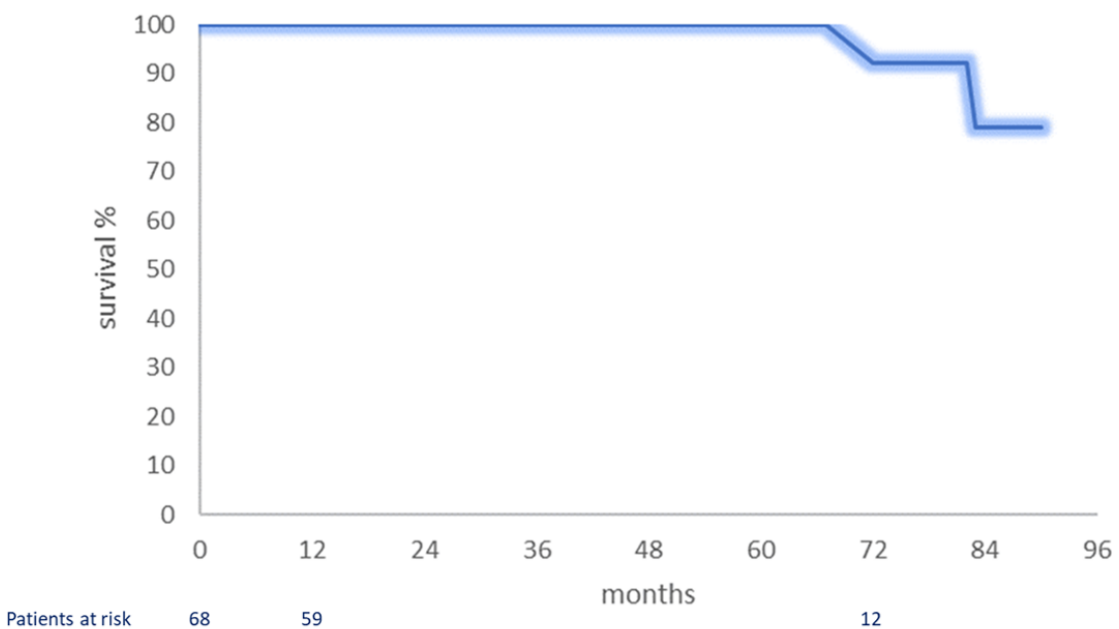

Figure 2. Kaplan-Meier curve freedom from mild Tricuspid regurgitation 


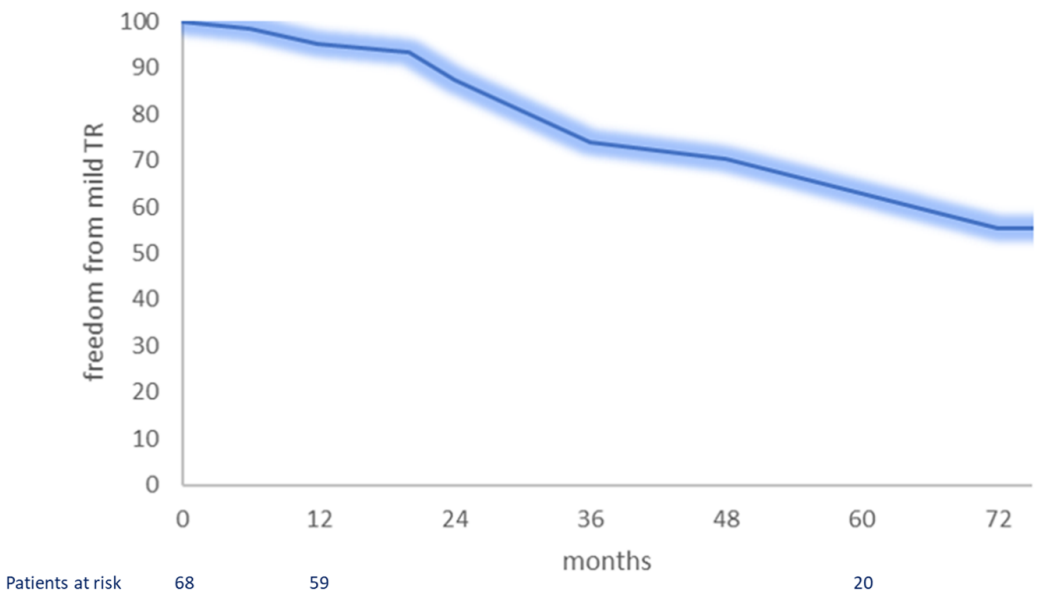

[1] Paparella D, Malvindi PG, Santarpino G, Moscarelli M, Guida P, Fattouch K et al. Full sternotomy and minimal access approaches for surgical aortic valve replacement: a multicentre propensity-matched study. Eur J Cardiothorac Surg 2020;57:709-16.

[2] Casselman FP, Van Slycke S, Wellens F, et al. Mitral valve surgery can now routinely be performed endoscopically. Circulation 2003; 108 Suppl 1:II48-54

[3] Modi P, Rodriguez E, Hargrove III C. Minimally invasive mitral valve surgery: a systematic review and meta-analysis. Eur J Cardio-thorac Surg. 2008; 34(5):943-952.

[4] Arman Kilic, Wilson Y. Szeto, Pavan Atluri, Michael A. Acker, W. Clark Hargrove. Operatiove Otcomes of Concomitant Minimally Invasive Mitral and Tricuspid Valve Surgery. Innovations. 2019, Vol. 14(5) 412-418]

[5] Bettina Pfannmuller, Piroze Davierwala, Gregor Hirnle, Michael A. Borger, Martin Misfeld, Jens Garbade, Joerg Seeburger, Friedrich W. Mohr Concomitant tricuspid valve repair in patients with minimally invasive mitral valve surgery. Ann Cardiothorac Surg 201; 2(6):758-764

[6] Davide Ricci, Massimo Boffini, Cristina Barbero, Suad El Qarra, Giovanni Marchetto, Mauro Rinaldi. Minimally invasive tricuspid valve surgery in patients at high risk. J Thorac Cardiovasc Surg 2014; 147:9961001

[7] Hector Cubero-Gallego, Maria Heredia-Rodriguez, Eduardo Tamayo. Perioperative myocardial infarction after heart valve surgery, where are we going? The Journal of Thoracic and Cardiovascular Surgery

[8] Grant SW, Hickey GL, Modi P, Hunter S, Akowuah E, Zacharias J. Propensity-matched analysis of minimally invasive approach versus sternotomy for mitral valve surgery. Heart. 2019 May;105(10):783-789

[9] Svensson LG, Atik FA, Cosgrove DM, Blackstone EH, Rajeswaran J, Krishnaswamy G, Jin U, Gillinov AM, Griffin B, Navia JL, Mihaljevic T, Lytle BW. Minimally invasive versus conventional mitral valve surgery: a propensity-matched comparison. J Thorac Cardiovasc Surg. 2010 Apr;139(4):926-32]

[10] Tang P, Onaitis M, Gaca JG, Milano CA, Stafford-Smith M, Glower D. Right Minithoracotomy Versus Median Sternotomy for Mitral Valve Surgery: A Propensity Matched Study. Ann Thorac Surg. 2015 Aug;100(2):575-81]

[11] Paparella D, Fattouch K, Moscarelli M, Santarpino G, Nasso G, Guida P, Margari V, Martinelli L, Coppola R, Albertini A, Del Giglio M, Gregorini R, Speziale G. Current trends in mitral valve surgery: A multicenter national comparison between full-sternotomy and minimally-invasive approach. Int J Cardiol. 2020 May 1;306:147-151] 
[12] Malvindi PG, Margari V, Mastro F, Visicchio G, Kounakis G, Favale A, Dambruoso P, Labriola C, Carbone C, Paparella D. External aortic cross-clamping and endoaortic balloon occlusion in minimally invasive mitral valve surgery. Ann Cardiothorac Surg. 2018 Nov;7(6):748-754

[13] Arman Kilic, Wilson Y. Szeto, Pavan Atluri, Michael A. Acker, W. Clark Hargrove. Operatiove Otcomes of Concomitant Minimally Invasive Mitral and Tricuspid Valve Surgery. Innovations. 2019, Vol. 14(5) 412$418]$

[14] Bettina Pfannmuller, Piroze Davierwala, Gregor Hirnle, Michael A. Borger, Martin Misfeld, Jens Garbade, Joerg Seeburger, Friedrich W. Mohr Concomitant tricuspid valve repair in patients with minimally invasive mitral valve surgery. Ann Cardiothorac Surg 201; 2(6):758-764

[15] Davide Ricci, Massimo Boffini, Cristina Barbero, Suad El Qarra, Giovanni Marchetto, Mauro Rinaldi. Minimally invasive tricuspid valve surgery in patients at high risk. J Thorac Cardiovasc Surg 2014; 147:9961001

[16] Murzi M, Kallushi E, Solinas M, Glauber M. Video-assisted right atrial surgery with a single two-stage femoral venous cannula. Interact Cardiovasc Thorac Surg. 2009 Jul;9(1):9-10]

[17] Murzi M, Miceli A, Di Stefano G, Cerillo AG, Farneti P, Solinas M, Glauber M. Minimally invasive right thoracotomy approach for mitral valve surgery in patients with previous sternotomy: a single institution experience with 173 patients. J Thorac Cardiovasc Surg. 2014 Dec;148(6):2763-8]

[18] Peng R, Ba J, Wang C, Lai H, Hu K, Shi H. A New Venous Drainage Technique in Minimally Invasive Redo Tricuspid Surgery: Vacuum-Assist Venous Drainage via a Single Femoral Venous Cannula. Heart Lung Circ. 2017 Feb;26(2):201-204]

[19] Sansone F, del Ponte S, Zingarelli E, Casabona R. Internal snaring of the caval veins by Foley catheters in case of reoperation via right thoracotomy. Interact Cardiovasc Thorac Surg. 2011 Oct;13(4):370-2

[20] Sansone F, Barbero C, Rinaldi M. Occlusion of both caval veins by an endovascular occluder. Heart Lung Circ. 2012 May;21(5):275-7]

[21] Dandolu R, Eaton D, Ali A, Schwann N, Wechsler A. Right atrial surgery without caval snaring. Innovations (Phila). 2005 Winter;1(2):75-8]. 